\title{
Light intensity enhancement inside the grooves of metallic gratings
}

\author{
Francisco Llopis, ${ }^{1, *}$ Ignacio Tobías, ${ }^{2}$ and Mario M. Jakas ${ }^{1}$ \\ ${ }^{1}$ Departamento de Fisica Fundamental y Experimental, Universidad de La Laguna, \\ Avenida Astrofisico Francisco Sanchez s/n, 38203 La Laguna, Tenerife, Spain \\ ${ }^{2}$ Instituto de Energla Solar, Universidad Politecnica de Madrid, ETSI Telecomunicacion, \\ 28040 Ciudad Universitaria, Madrid, Spain \\ *Coresponding author: fllopisioull.es
}

\begin{abstract}
Light absorption inside the grooves of metallic gratings filled with a semiconductor material can be improved by means of the electric field enhancement. To this end, the influence of grating dimensions in the electric field spectral behavior is theoretically investigated. Two conditions of cavity resonance have been analyzed separately: (1) for TE polarization (electric field parallel to the grooves) and (2) for TM polarization (magnetic field parallel to the grooves). When dimensions are chosen acoording to the first condition, the enhancement of TE fields is found to increase with the height to width ratio, and it is accompanied with a decrease in the bandwidth. The same enhancement levels can be achieved for TM fields if the second condition holds, provided that the period to width ratio is large enough. The simultaneous enhancement of TE and TM fields, based on a condition of surface resonance excitation, can also be accomplished. In this case, the TM response is very sensitive to changes in groove depth and width. $(2010$ Optical Society of America

OCIS codes: $050.2770,260.5740,350.6050$.
\end{abstract}

\section{INTRODUCTION}

Light absorption in solar cells must be increased as much as possible to enhance the conversion efficiency. For instance, prototype intermediate-band solar cells (IBSCs) fabricated with quantum-dot (QD) absorbers feature low absorbance for infrared wavelengths [1]. Incomplete absorption also affects the performance of thin film silicon solar cells [2]. On the other hand, upconversion effects that have been proposed to make use of sub-bandgap wavelengths in silicon need high electromagnetic densities to be efficient [3].

Light trapping is successfully used in silicon solar cells to enhance absorption of near-bandgap light. It relies on producing rough surfaces, normally textured with micronsized features, larger than the confined wavelengths that randomize the ray directions in the substrate so that light is trapped by total internal reflection. Absorption is enhanced by a factor of $40-50$ at maximum in a wide wavelength range and without dependence on the incoming light direction $[4,5]$. This approach is not easily applied to thin film solar cells.

Absorption is proportional to the squared amplitude of the electric field $[6,7]$. Very strong enhancement of the local electric field can be obtained by capitalizing on resonance phenomena in the near field: surface plasmons in metal nanoparticles $[8,9]$ or confined resonances in metallic gratings can both increase the electric field and boost the rate of optical processes in matter. This approach has been implemented for surface enhanced Raman scattering [10], which involves both absorption and emission, and hence its strength increases as the fourth power of the electric field.
In this paper, we consider the electromagnetic resonances taking place in metallic diffraction gratings as a means of electric field enhancement and improved absorption in solar cells. As recently reported, the sensitivity of a QD photodetector improves if the active layer is located under a metallic strip grating [11]. But in photovoltaic applications it is important to take into account that absorption must be increased in a large volume, in a wide bandgap range and for incident light within a large interval of directions and with random polarization. Although special reference will be made to IBSCs, we think that the approach can be of interest for thin cells or for upconversion schemes based on atomic transitions (in this case, since two photons are generally involved the upconversion rate increase will be stronger).

Electric field enhancement in metallic diffraction gratings has been studied for both TE and TM polarizations. A number of groups have addressed the different nature of resonances and their dependence on structural and material properties [12-17]. Different methods are available for calculating the response of a diffraction grating. For lamellar gratings, an analytical approach based on expansion in waveguide modes has been used. This method is readily applied for perfect conductors or, under certain conditions, real conductors by incorporating approximate boundary conditions at the dielectric-metal interface.

In this paper we study the electric field enhancement in perfect conductor one-dimensional metallic gratings. We begin by putting forward the modal method for calculating the response of a metallic grating. Then we study the field enhancement for TE polarized light in the vicinity of the cavity resonances taking place in the grooves and the 
dependence with wavelength and aspect ratio. The case of TM polarization is considered next. We end with an assessment of the potential of metallic gratings for photovoltaic devices.

As mentioned before, the excitation of surface plasmons can also contribute to the field enhancement. For this purpose, the diffraction grating can provide the extra momentum required to excite the collective oscillations of the surface electronic density. However, this situation is not discussed in this study since the perfect conductor model does not incorporate the metal-dielectric functions. This mechanism is not considered in this paper given that we intentionally focus our attention on the ideal cavity resonances themselves. In this sense, the values obtained for field enhancement must be quoted as upper limits.

\section{OUTLINE OF THE THEORY}

\section{A. Problem Statement}

In this subsection we summarize the main aspects of the modal method [12-19] with reference to the situation depicted in Fig. 1. A perfectly conducting diffraction grating with groove spacing $d$ is exposed to a monochromatic TE polarized wave with a vacuum wavelength $\lambda$. The groove depth and aperture size are $h$ and $c$, respectively. The whole structure is surrounded by a non-conducting medium with a refractive index $v_{1}$. The incident electric field is assumed to have unit amplitude and can be expressed as

$$
E_{2 i}(x, y)=\exp \left[i\left(\alpha_{i} x-\chi_{10} y\right)\right]
$$

where the components of the incident wavevector are given by

$$
\alpha_{0}=\kappa \nu_{1} \sin \theta, \quad \chi_{0}=\kappa \nu_{1} \cos \theta,
$$

with $\theta$ being the incidence angle and $\kappa=2 \pi / \lambda$ being the vacuum wavenumber.

The interaction between the incoming wave and the grating results in an electric field distribution inside the grooves that can be expressed in terms of cavity modes. On the other hand, the total electric field above the grating can be expressed in terms of the plane wave expansion

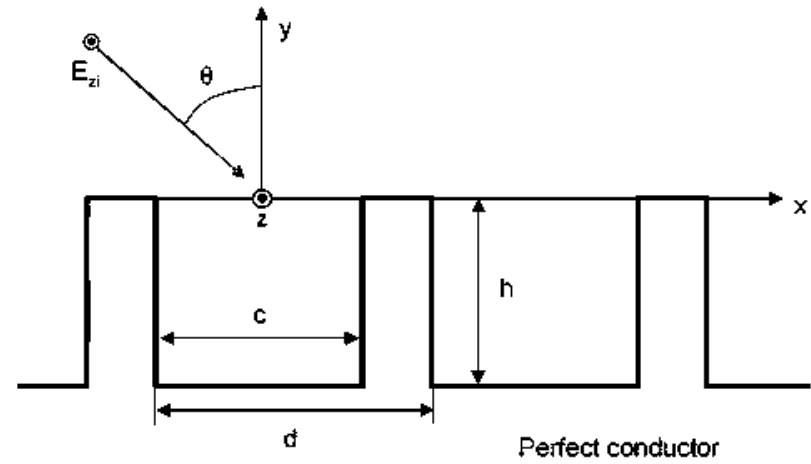

Fig. 1. Perfectly conducting grating illuminated by a TE polarized wave.

$$
E_{R}(x, y)=E_{z i}(x, y)+\sum_{n=-\infty}^{\infty} A_{n} \exp \left[i\left(\alpha_{n} x+\chi_{n} y\right)\right]
$$

where the $n$th reflected wave has an amplitude $A_{n}$ and wavevector components

$$
\alpha_{n}=\alpha_{i)}+n \frac{2 \pi}{d}, \quad \chi_{n}=\sqrt{\kappa^{2} v_{1}^{2}-\alpha_{n}^{2}} .
$$

Each reflected wave is said to be propagating if $\chi_{n}$ is positive real, or evanescent, assuming that $\chi_{n}$ is positive imaginary in the last case. For a perfectly conducting grating, energy conservation constraint implies

$$
\sum_{n=N_{1}}^{N_{2}}\left|A_{n}\right|^{2} \frac{\chi_{n}}{\chi_{0}}=1
$$

where the summation extends only to non-evanescent orders $n=N_{1}, \ldots, N_{2}$.

Fields inside the grooves satisfy the wave equation

$$
\nabla^{2} E_{z}+\kappa^{2} v_{1}^{2} E_{z}=0
$$

Since the electric field must vanish at the groove walls $(x= \pm c / 2)$ and at the bottom $(y=-h)$, separation of variables allows us to express the electric field inside each groove by means of a modal expansion [12],

$$
E_{M}(x, y)=\sum_{m=1}^{\infty} a_{m} \phi_{m}(x, y)
$$

where

$$
\phi_{m}(x, y)=\sin \left[\mu_{m}(y+h)\right] \sin \left(\frac{m \pi x}{c}\right) \text { if } m \text { is even, }
$$

$$
\phi_{m}(x, y)=\sin \left[\mu_{m}(y+h)\right] \cos \left(\frac{m \pi x}{c}\right) \text { if } m \text { is odd }
$$

with

$$
\mu_{m}=\sqrt{\left(\frac{2 \pi \nu_{1}}{\lambda}\right)^{2}-\left(\frac{m \pi}{c}\right)^{2}}
$$

being the separation constant, which is positive (either a real or imaginary number). Once the modal and reflectedorder amplitudes are known, we can analyze the electric field behavior inside the cavities, as well as the near field behavior above the grating. Further theoretical foundations, based on the application of the modal method to calculate the amplitudes, are given in the Appendix. The procedure can be extended to the case of TM polarization after replacing the electric fields with their magnetic field counterparts $H_{z}$ and $H_{z i}$ in Eqs. (3) and (6); but in this case the series in Eq. (6) runs from $m=0$, and modal functions are the same as those in Eqs. (7) and (8) with sines and cosines interchanged. If the diffraction grating is illuminated by unpolarized light, we can obtain an estimate of the electric field enhancement by averaging the squared amplitudes of the TE and TM fields inside the grooves. 


\section{B. Resonant Enhancement of the Electric Field for TE Polarization}

Each metallic groove can enhance the internal electric or magnetic fields if some kind of constructive interference is induced, giving as a result a standing wave pattern. In this subsection we will examine how this situation can be achieved for certain wavelengths and the degree of enhancement of the internal fields for TE polarization.

Since the incoming and reflected plane waves are coupled with cavity modes, the unknown amplitudes can be obtained by the proper matching of the tangential electric and magnetic fields through the aperture. Boundary conditions at the metallic top facets must also be taken into account. In this manner, we can derive a coupled system with the modal and diffracted wave amplitudes as unknowns.

Alternatively $[13,14]$, this coupled system can be recast into a matrix equation $\mathbf{G a}=\mathbf{H}$, with a being the vector of modal amplitudes (see the Appendix for details). As previously said, the grating dimensions must be chosen to enhance the electric field through the excitation of cavity resonances. Whether or not the enhancement is feasible depends on the spectral behavior of the $\mathbf{G}$ matrix since zeros of $\operatorname{det}(\mathbf{G})$ are linked to the electric field resonances inside the grooves. Therefore, for a given set of parameters $(\theta, h, c, d)$ we could study the behavior of $\operatorname{Re}[\operatorname{det}(\mathbf{G})]$ and $\operatorname{Im}[\operatorname{det}(\mathbf{G})]$ to search for the wavelength range for which the field is enhanced. But, in accordance with Wirgin and Maradudin [13,14], the resonance conditions can be stated in a more efficient way for normal incidence if:

(1) grating operates in the monomode regime. Hence, the electric field inside the grooves is dominated by the fundamental mode $(m=1)$. The remaining modes have an imaginary $\mu_{m}$ spatial frequency and negligible amplitude. This condition is achieved for wavelengths comprised between $c v_{1}$ and $2 c \nu_{1}$.

(2) the dominant reflected orders are the specular one (zeroth order) and \pm 1 st orders; these last are assumed to be evanescent. Therefore, the condition $\lambda>d \nu_{1}$ must hold.

Under these assumptions, Wirgin and Maradudin $[13,14]$ found that zeros of $\operatorname{Re}[\operatorname{det}(\mathbf{G})]$ and $\operatorname{Im}[\operatorname{det}(\mathbf{G})]$ are, respectively, close to zeros $\lambda^{R}$ and $\lambda^{I}$ of

$$
\begin{gathered}
D^{R}(\lambda)=\mu_{1}(\lambda)+4 \frac{\mathcal{c}}{d}\left|\chi_{1}(\lambda)\right|\left|J_{11}(\lambda)\right|^{2} \tan \left[\mu_{1}(\lambda) h\right], \\
D^{I}(\lambda)=2 \frac{c}{d} \chi_{10}(\lambda)\left|J_{10}(\lambda)\right|^{2} \tan \left[\mu_{1}(\lambda) h\right]
\end{gathered}
$$

where $J_{10}(\lambda)$ and $J_{00}(\lambda)$ stem from the coupling between $n=0, \pm 1$ diffracted orders and the $m=1$ mode. In general, the coupling coefficients $J_{m n}(\lambda)$ are computed as (see the Appendix)

$$
J_{m n}(\lambda)=\frac{1}{c} \int_{-c / 2}^{c / 2} \exp \left[i \alpha_{n}(\lambda) x\right] \cos \left(\frac{m \pi x}{c}\right) \mathrm{d} x .
$$

The resonance wavelengths are located inside the intervals $\left[\lambda_{j}^{I}, \lambda_{j}^{R}\right]$, with $j=1,2, \ldots$. In particular, $\lambda_{j}^{I}$ roots can be calculated in closed form as

$$
\lambda_{j}^{I}=2 \nu_{1}\left(j^{2} / h^{2}+1 / c^{2}\right)^{-1 / 2}, \quad j=1,2 \ldots .
$$

Thus, each resonant wavelength is close to a quasiresonant wavelength $\lambda_{j}^{I}$ related to the groove shape but not to the groove spacing $d$. As we will see later, Eq. (12) provides a useful criterion when selecting a suitable $h / c$ aspect ratio to achieve the desired resonance wavelength. This reveals that the resonance takes place inside the groove with little influence of neighboring ones. In fact, it expresses that the tangential electric field in the $y=0$ plane is zero, as for a metallic resonator.

The assumptions made herein have proven to be useful to seek the features of a structure intended to enhance the internal TE electric field. Although we will comment on the results in the next section, it is interesting to conclude this section analyzing the amplitude of the enhancement. We can pay attention to the behavior of the field at the line $x=0$. If the conditions stated above still hold, the magnitude of the electric field inside the grooves at the quasi-resonant wavelength $\lambda_{j}^{I}$ (see the Appendix) can be expressed as

$$
|E(0, y)|_{\lambda=\lambda_{j}^{I}}=\frac{8 \chi_{0}}{\pi}\left|\frac{\sin \left[\mu_{1}\left(\lambda_{j}^{I}\right)(y+h)\right]}{\mu_{1}\left(\lambda_{j}^{I}\right)}\right| .
$$

Thus, the electric field magnitude, for a given value of $y$, is an oscillatory function of $\mu_{1}$ that achieves its absolute maximum for $\mu_{1}=0$. As appointed in $[13,14]$, the magnitude will be larger the smaller is $\mu_{1}\left(\lambda_{j}^{I}\right)$, which in turn implies to select $h$ as large as possible. In the limit $h \rightarrow \infty$ we find $\lambda_{j}^{I}=2 v_{1} c$.

\section{Resonant Enhancement of the Electric Field for TM Polarization}

In photovoltaic applications, the behavior of the structure when exposed to a TM polarized wave must also be considered. Let the magnetic fields inside the grooves be dominated by the fundamental mode contribution, whose amplitude $b_{0}$ reads $[16,17]$

$$
b_{0}=\frac{2 \operatorname{sinc}\left(\alpha_{(1)} / 2\right)}{\sin \left(\mu_{(0} h\right)} \frac{1}{\left.\cot \left(\mu_{(0} h\right)-i \mu_{0}\right) \frac{c}{d} \sum_{n=-\infty}^{\infty} \frac{\left[\operatorname{sinc}\left(\alpha_{n} c / 2\right)\right]^{2}}{\chi_{n}}}
$$

where $\mu_{0}=\kappa \nu_{1}=2 \pi \nu_{1} / \lambda$ and $\operatorname{sinc}(z)=\sin (z) / z$. The cavity modes $m=1,2, \ldots$ are evanescent if the condition $\lambda$ $>2 c \nu_{1}$ is satisfied. The resonance wavelengths are now found to be very close to zeros of $\cos \left(\mu_{0} h\right)[16,17]$. The modal fields have no $x$ dependence for the fundamental mode and read

$$
\begin{gathered}
H_{z}=b_{0} \cos \left[\mu_{0}(y+h)\right], \quad E_{x}=-\frac{i}{\omega \nu_{1}^{2}} b_{0} \mu_{0} \sin \left[\mu_{0}(y+h)\right], \\
E_{y}=0 .
\end{gathered}
$$

Under normal incidence, $\alpha_{0}=0$ and $\mu_{0}=\chi_{0}=\kappa \nu_{1}$. For wavelengths such that $\cos \left(\mu_{0} h\right)=0$, we also have $\sin \left(\mu_{0} h\right)=1$ and $\cot \left(\mu_{0} h\right)=0$. By further assuming that the series can be replaced with the $n=0$ th term, we can calculate the amplitude of the fundamental mode as $b_{0}=2 i(d / c)$. For 
these wavelengths, the squared magnitude of the electric field at $(0,-0.5 h)$ is enhanced by a factor $2\left(d^{2} / c^{2}\right)$ with respect to that of the incident field $[15,17]$.

The condition that the cosine is null corresponds again to the tangential electric field becoming zero at the $y=0$ interface. However, this is not always accompanied by a large increase in the electric field, as we will notice later. For TM polarization, there are both cavity and surface resonances $\left(d=\lambda_{r} / \nu_{1}\right)$ that may interact with each other, and the situation is more complex than for TE resonances [16]. We will also consider the possibility of simultaneous enhancement for both polarizations employing this criterion.

\section{RESULTS}

In this section we present the results of calculating the electric field inside the grooves. Although the grating dimensions have been chosen following the criteria suggested in Subsections BC2 and 2, the calculations have been performed by matrix inversion. Occasionally, results based on the approximate models have also been shown for the sake of comparison.

\section{A. Application of the Griterion for TE Field Resonant Enhancement: Choice of Grating Dimensions and Spectral Behavior}

Let us assume that the structure we are dealing with enhances the internal fields if illuminated with light capable of producing produce subband transitions. Specifically, QD layers currently designed for AsGa IBSCs are sensitive to photon energies about $0.3 \mathrm{eV}$. We can thus pick the dimensions of the grating intended to exhibit a resonance wavelength $\lambda_{r}$, which can be calculated using the expression $\lambda_{r}[\mu \mathrm{m}]=1.24 / \varepsilon_{r}[\mathrm{eV}]$, with $\varepsilon_{r}$ being the photon energy. Given the $h / c$ ratio and the wavelength $\lambda_{r}$, we can calculate the groove width $c$ from Eq. (12). As was previously mentioned, however, if the groove depth is large enough, $c$ can also been obtained by using the expression $c=\lambda_{r} / 2 \nu_{1}$. This latter choice produces $c$ $=0.57 \mu \mathrm{m}$ for $\varepsilon_{r}=0.3 \mathrm{eV}$ and $\nu_{1}=3.6$, i.e., the refractive index of AsGa at $0.3 \mathrm{eV}$. The groove spacing $(d)$ and depth (h) are the remaining parameters. Before discussing the influence of the $d / c$ ratio, we first select $d=1.1 c$. Since a deep enough groove must be chosen to fulfill the optimal condition for the electric field, we assume $h \geq 2 c$. Finally, given that photovoltaic cells of concentration systems are exposed to a bundle of rays, the optical response of the grating in the case of conical light incidence should be considered. However, for concentration levels of 500 suns or even greater, the angle of the illuminating conic bundle is about $10^{\circ}$. Therefore, we have considered only the case of normal incidence.

As shown in Fig. 2, the squared magnitude of the electric field achieves its maximum close to the predicted resonant wavelength, even for the shallowest grating. For greater aspect ratios, and within the range considered, the plots exhibit secondary peaks, in agreement with Eq. (12). The same figure includes, in an enlarged view, the plot for $h / c=4$ around the first resonance peak. The interval limits in terms of photon energy, 0.305 and $0.309 \mathrm{eV}$, are also sketched. These are related to the solutions of

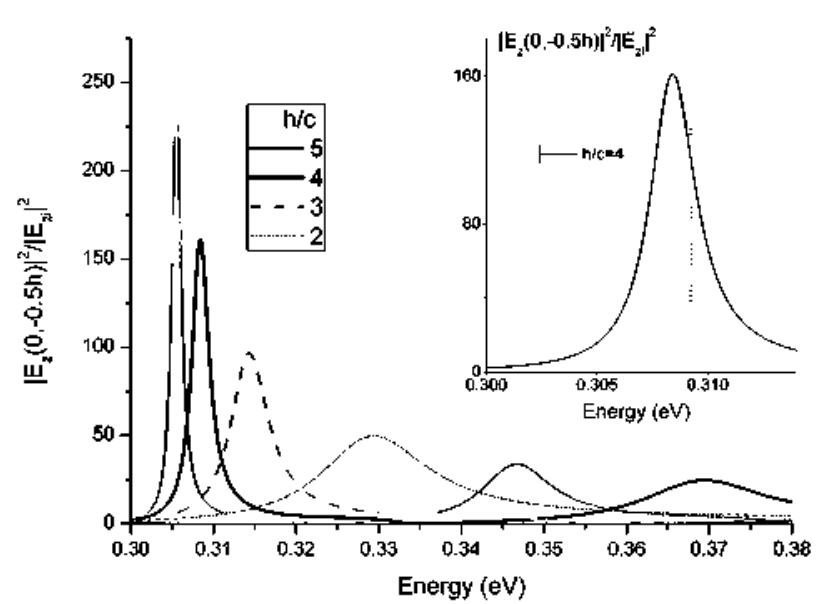

Fig. 2. Plots of the squared magnitude of the normalized electric field for $d=1.1 c$ and different depth to width ratios. The inset shows an enlarged view of the plot for $h / c=4$ near the maximum of the normalized electric field $(\approx 0.308 \mathrm{eV})$.

Eqs. (10) and (11) for $j=1$. At least for TE polarization, it seems that the estimated intensity peak location does not roughly depart from our objective. Notice that, by properly changing the grating dimensions, these plots can be shifted slightly down toward lower energies in order to achieve the maximum at the required wavelength. This issue will be explored afterward.

Efficient utilization of the solar spectrum would require the absorption to be as wide as possible. However, the spectral width of the transitions can be much smaller if they take place through localized levels, as in intermediate-band or upconversion materials. At any rate, and depending on the application, the selectivity of the structure must be taken into account so that it matches the absorption features of the material. Therefore, we can calculate the spectral bandwidth of the main resonances in terms of the full width at half-maximum (FWHM) of each intensity profile. In order to asses the influence of the period to aperture size ratio $(d / c)$ in both FWHM and intensity peaks, we have carried out a parametric analysis. The spectral bandwidth, as depicted in Fig. 3 , decreases with the $h / c$ ratio, in agreement with the profile broadening observed in Fig. 2. Therefore, we can

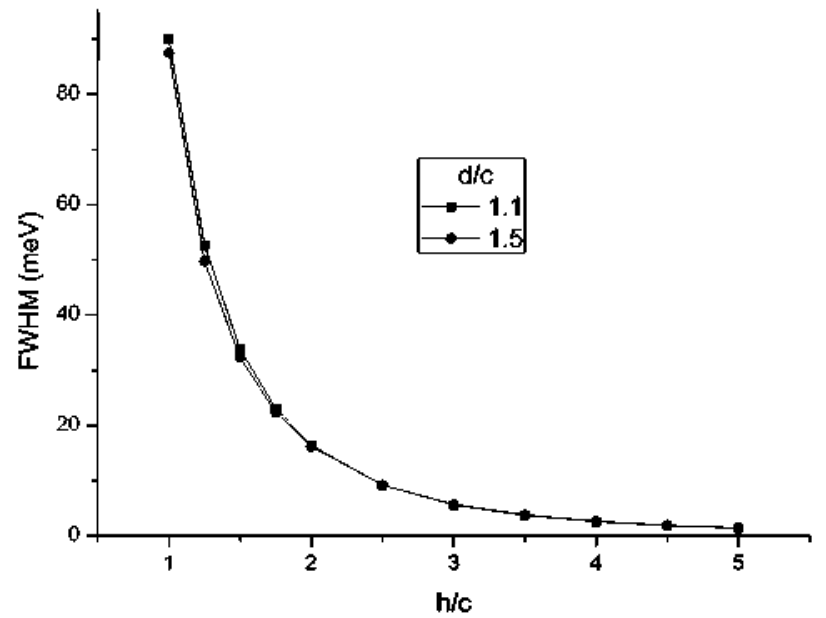

Fig. 3. FWHM as a function of $h / c$ ratio, where $h$ and $c$ are the depth and width of the grooves, respectively. 
conclude that for the selection of the structure dimensions the trade-off between the achievable intensity and bandwidth must not be disregarded. For example, in order to attain a bandwidth above the $10 \mathrm{meV}$ threshold, ratios $h / c$ greater than 2 appear to be suitable for a ratio of $d / c=1.1$. The FWHM remains insensitive to the $d / c$ ratio for the whole $h / c$ range. Since plots are nearly the same for $d / c$ ranging from 1.1 to 1.5 , only results for these extreme cases are depicted.

The peak values of the squared electric field, namely, $\left|E_{z_{p}}\right|^{2}$, increase with the $h / c$ ratio, as shown in Fig. 4, and do not strongly depend on the $d / c$ ratio for the shallower gratings (i.e., with $h / c<2$ ). It must be noticed that the resonance energy does not seem to depend on $d / c$. Actually, only for $h / c<2 \varepsilon_{r}$ appears to be a slightly increasing function of $d / c$. As one can see in Fig. 5, $\varepsilon_{r}$ increases with a decrease in $h / c$ and approaches asymptotically to the theoretical value of $0.3 \mathrm{eV}$ given by Eq. (12) for large $h / c$ values. In Fig. 6 we have plotted the previously calculated field enhancements times the corresponding FWHM. The products so obtained are decreasing functions of $h / c$; and, except for one point that was carefully checked for artifacts, they all seem to be a slightly increasing function of $d / c$. All these results indicate that for TE polarization, both resonance intensities and bandwidths are to a large extent controlled by the aspect ratio $h / c$, whereas the $d / c$ ratio is a rather less important parameter, becoming slightly relevant only for groove depths larger than, say, $2 c$.

Despite that the perfect conductor grating can enhance the internal TE polarized electric field, the arrangement has a poor response to TM polarized waves. The intensities can now attain lower levels, indeed, because the condition of resonance is quite different, as indicated in Subsection 2.C. As shown in Fig. 7, if groove dimensions are chosen according to the criterion stated in the preceding paragraphs, it cannot obtain a noticeable enhancement. Furthermore, the electric field vanishes for certain $h / c$ aspect ratios for $\lambda=\lambda_{r}$. This behavior can be easily explained since, for the cases considered, the condition $\lambda_{r}>2 c \nu_{1}$ holds. Thus, the magnetic field is dominated by the fundamental mode at the resonant wavelength. Therefore,

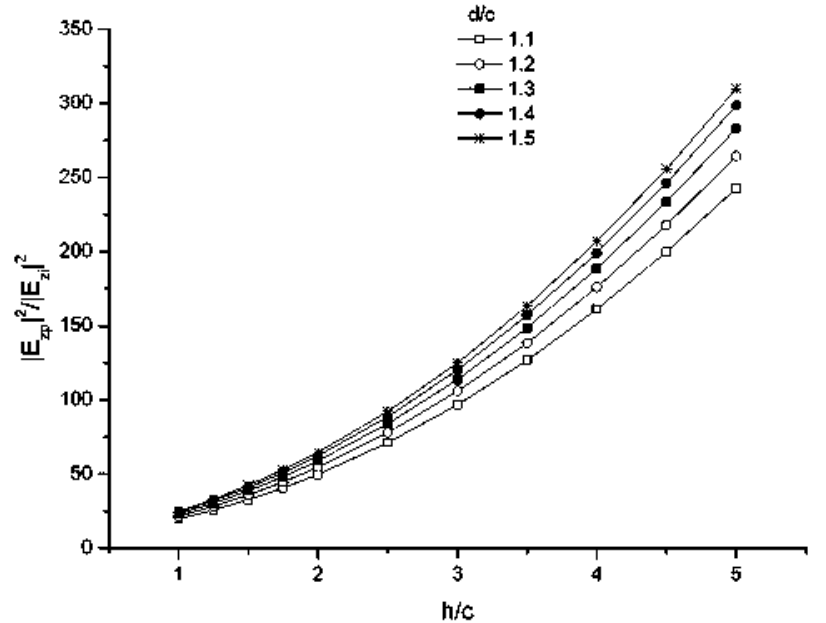

Fig. 4. Maximum intensity versus $h / c$ ratio for different values of $d / c$ ratio, where $d$ is the period of the grating and $c$ is the width of the grooves.

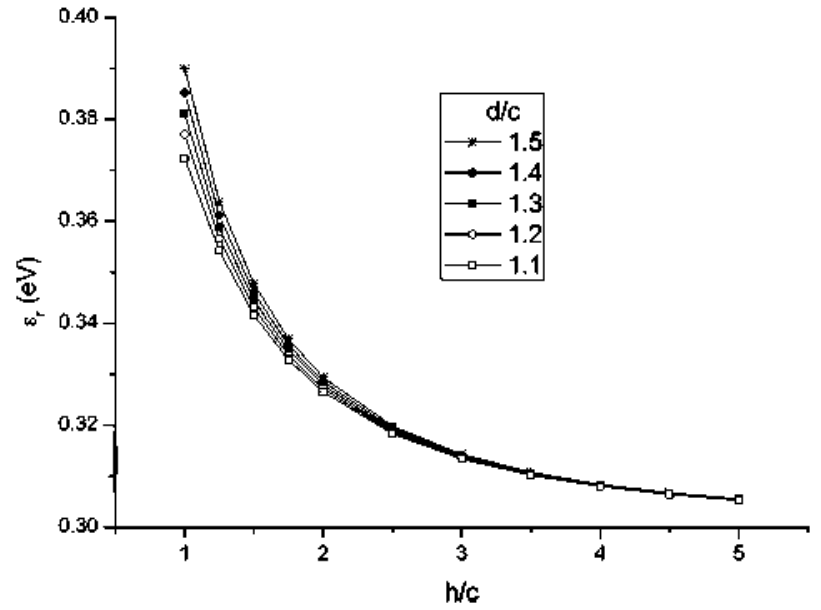

Fig. 5. Resonance energies versus $h / c$ ratio for different values of $d / c$ ratio.

we find $E_{y}=0$ and $E_{x}=$ const $\times \sin \left[\mu_{0}(y+h)\right]$. Setting $h=n c$, where $n$ is an integer, and choosing again $c$ $=\lambda_{r} / 2 \nu_{1}$, the TM $E_{x}$ field at $y=-0.5 h$ is proportional to $\sin \left(n \pi \lambda_{r} / 2 \lambda\right)$, which vanishes at the resonance wavelength when $n$ is even. However, although $E_{x}$ does not vanish for $\lambda=\lambda_{r}$, when $n$ is odd, a great enhancement is not observed either. This fact indicates that, after averaging the TE and TM intensities, the enhancement will be reduced to a value around one half of the TE polarization maximum.

As previously said, the resonance wavelengths can be shifted toward our target after multiplying the spatial dimensions by a factor $f$. The cavity-mode frequencies and reflected-order wavenumbers are thereby scaled by a factor $1 / f$. Therefore, neither the phases of the incident and reflected fields nor the modal functions change after the scaling, and the solution of the boundary value problem remains the same. Of course, the invariance would fail if the refractive index $\nu_{1}$ depends on the wavelength (which is assumed to have a constant value in this paper). Figure 8 illustrates the effect of the scaling. With a ratio of $d / c$ $=1.1$, in our first attempt, the peaks were located at 0.306 $\mathrm{eV}$ (for $h / c=5$ ) and $0.308 \mathrm{eV}$ (for $h / c=4$ ). In order to place

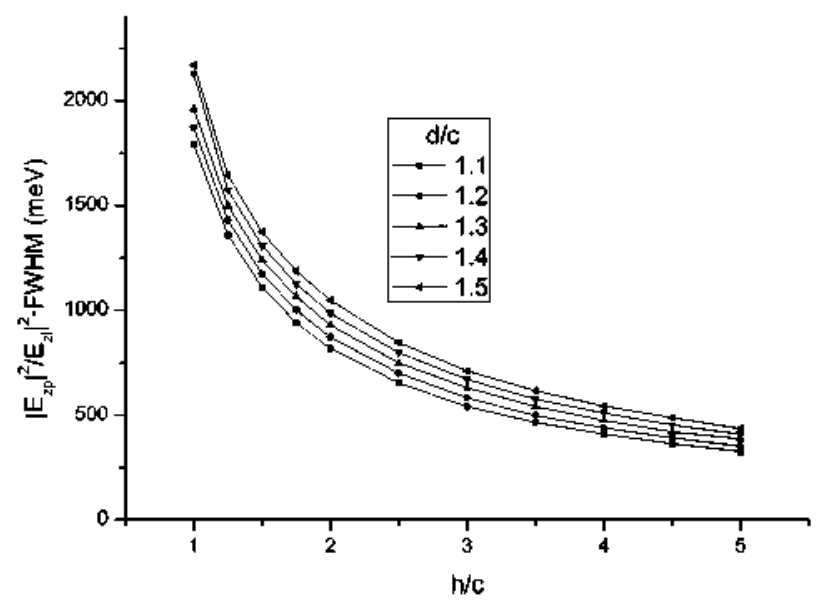

Fig. 6. Field enhancement-bandwidth product, i.e., $\left|E_{z p}\right|^{2} /\left|E_{z p}\right|^{2}$ $\times$ FWHM, for structures with different $d / c$ ratios in terms of the $h / c$ ratio (see text). 

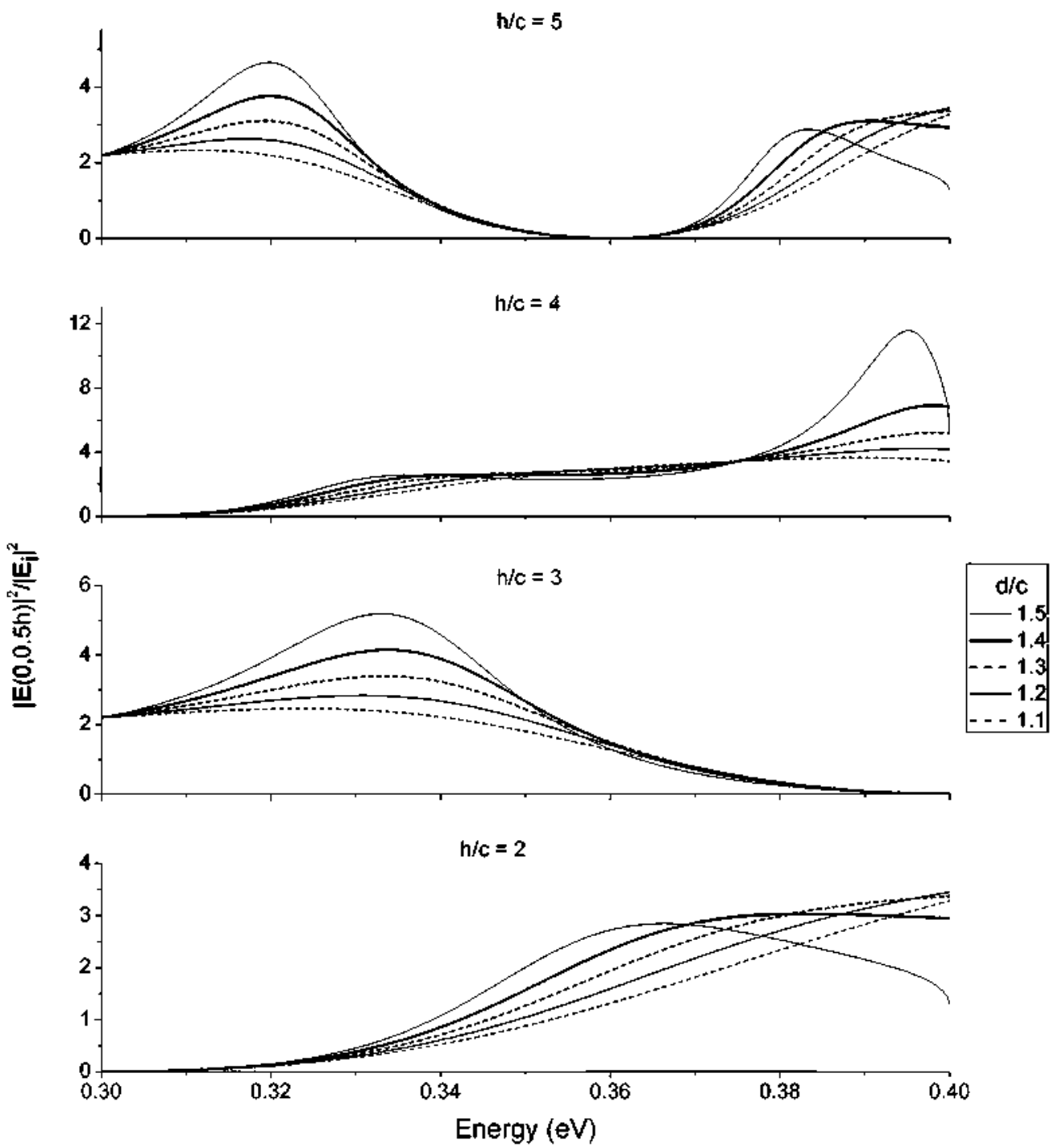

Fig. 7. Application of the criterion for finding TE field resonant enhancement: squared magnitude of the TM normalized electric field (see Subsection 3.A).

the peaks at $0.3 \mathrm{eV}$, it suffices to apply scaling factors $f_{1}$ $=0.306 / 0.3=1.02$ and $f_{2}=0.308 / 0.3=1.03$.

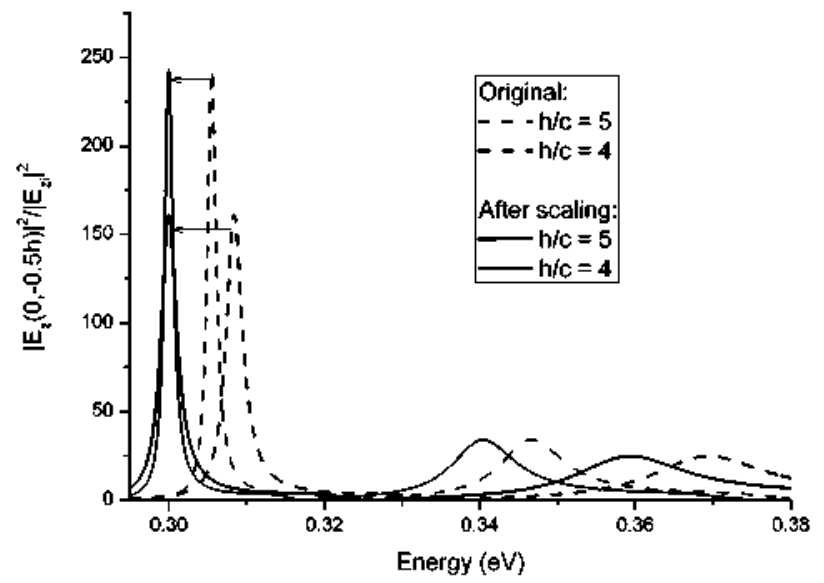

Fig. 8 . Shifting the resonance energy by changing the groove dimensions acxording to the scaling proposed in Subsection 3.A for aspect ratios $h / c=4$ and 5 . In both cases, a ratio $d / c=1.1$ is assumed.

\section{B. Application of the Criterion for TM Field Resonant Enhancement}

The second criterion results in a groove depth of $h$ $=\pi / 2 \mu_{0}=\lambda_{r} / 4 \nu_{1}=0.29 \mu \mathrm{m}$ for a resonance energy of 0.3 eV. The peak values of the squared TM electric fields at $(0,-0.5 h)$ are denoted now as $\left|\boldsymbol{E}_{p}\right|^{2}$, and their normalized values are depicted in Fig. 9. The upper limit values, i.e., the factors $2\left(d^{2} / c^{2}\right)$ mentioned in Subsection 2.C, are also shown. The bandwidths (not shown) are greater than those achieved previously for the TE field case. But peaks are of the same order only when the $d / c$ ratio is above 4, which is achieved only with very narrow grooves. Such a solution turns out to be a severe constraint for photovoltaic applications, in which photoabsorption is critical, since the available volume for the active layer is diminished.

\section{Simultaneous Enhancement of TM and TE Polarized Electric Fields}

Since the approaches followed herein can only be useful to establish the resonance wavelengths and spectral band- 


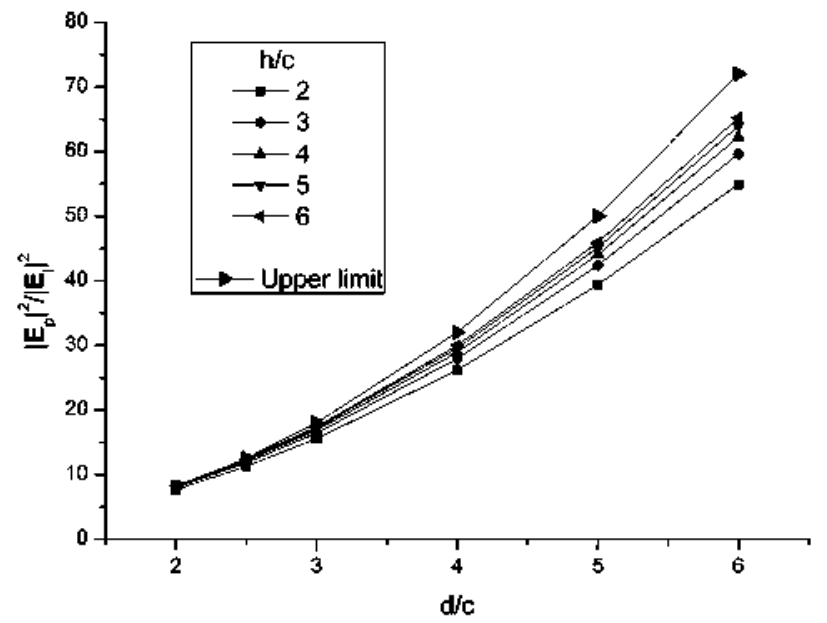

Fig. 9. Application of the criterion for finding TM field resonant enhancement: maximum intensity of the TM electric field in terms of $d / c$ ratio for different values of $h / c$ ratio.

widths for TE or TM electric fields independently, we have considered the third of the aforementioned criteria. If a surface resonance of the magnetic field is excited, a simultaneous resonance can be excited inside the grooves. This can be accomplished by choosing $d=\lambda_{r} / \nu_{1}$. In this manner, the period can be estimated from the resonant wavelength, with $c$ and $h$ being the remaining parameters. In order to assess the usefulness of this criterion, we have calculated the averaged response of the grating by performing a double sweep of both parameters. There are combinations for which the average is about 100 , but these extend over a narrow region as Fig. 10 reveals. Although not shown, the TM electric field peaks and their location are very sensitive to small changes in $c$ and $h$.

\section{SUMMARY}

The enhancement of the electric field inside the grooves of perfectly conducting gratings can be accomplished for both TE and TM polarizations. For TE polarization, a sig-

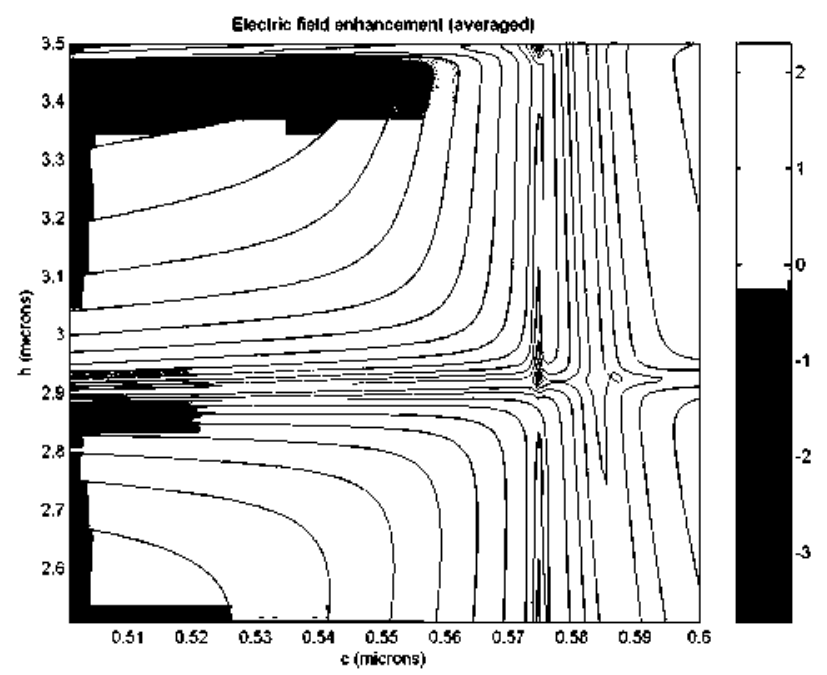

Fig. 10. Behavior of the (modulus squared) electric field at ( 0 , $-0.5 h)$ as a function of $h$ and $c$ and assuming $d=\lambda_{r} / \nu_{1}$ : the TE and TM responses have been averaged (a logarithmic scale is employed). nificant increase can be obtained inside the grooves. The wavelength at which it takes place is well predicted by Wirgin and Maradudin's criterion $[13,14]$ and depends on the groove width and depth. The slight deviations between predicted and real resonance wavelengths can be corrected by scaling. A possible drawback is the limited spectral bandwidth. It has been found that the higher the groove height to width ratio, the more intense the electric field and the narrower the resonant spectrum. The maximum enhancement-spectral width product is seen to decrease for deeper profiles.

This first criterion does not work for TM polarization, for which no field enhancement, or even suppression, is obtained. A second criterion has been stated by LópezRios et al. [16] for resonance of the TM polarization that is not compatible with the first one and, besides, does not always lead to larger electric fields inside the grooves. For this reason, we have performed a parametric analysis to find the ranges for which the electric fields are enhanced for both polarizations. Results show that there are parameter combinations for which the TE and TM electric fields can be simultaneously enhanced, but these occupy a very narrow region. We can conclude, thus, that the problem of the sensitivity to incident light polarization cannot be overcome at all.

The present work can be extended to two directions. First, we have focused our study on the limits that the different figures of merit can achieve in the ideal case. For non-ideal metal gratings the enhancement can drop significantly if conductors exhibit great losses for the wavelengths of interest. On the other hand, the behavior of three-dimensional symmetric crossed gratings can also be studied in order to ascertain if (1) the field enhancement increases and (2) the sensitivity to light polarization can be avoided.

\section{APPENDIX:}

We derive now some of the results aforementioned involving TE polarization. The tangential magnetic field must be continuous across the aperture of the diffraction grating. The electric field must be continuous across the aperture $(y=0,-c / 2 \leq x \leq c / 2)$ and vanish on the horizontal facets located at $(y=0, c / 2 \leq x \leq d / 2)$ and $(y=0,-d / 2 \leq x$ $\leq-c / 2$ ). Thus

$$
\begin{gathered}
\left(\frac{\partial E_{M}}{\partial y}\right)_{y=0^{-}}=\left(\frac{\partial E_{R}}{\partial y}\right)_{y=0^{+}}, \quad-c / 2 \leq x \leq c / 2, \\
E_{R}\left(x, 0^{+}\right)=E_{M}\left(x, 0^{-}\right), \quad-c / 2 \leq x \leq c / 2, \\
E_{R}\left(x, 0^{+}\right)=0, \quad-d / 2 \leq x \leq-c / 2, \quad c / 2 \leq x \leq d / 2 .
\end{gathered}
$$

Equation (A1) is projected onto the orthogonal set $\left\{\phi_{m}(x, 0),-c / 2 \leq x \leq c / 2\right\}$,

$\frac{1}{c} \int_{-c / 2}^{c / 2}\left(\frac{\partial E_{M}}{\partial y}\right)_{y=0^{-}} \phi_{m}(x, 0) \mathrm{d} x=\frac{1}{c} \int_{-c / 2}^{c / 2}\left(\frac{\partial E_{R}}{\partial y}\right)_{y=0^{+}} \phi_{m}(x, 0) \mathrm{d} x$,

giving, after some algebra, 


$$
\frac{1}{2} a_{m} \mu_{n} \cos \left(\mu_{n} h\right)=-i \chi_{(N} J_{m i)}+\sum_{n=-\infty}^{\infty} i \chi_{n} J_{m n} A_{n}
$$

with

$$
\begin{aligned}
& J_{m n}=\frac{1}{c} \int_{-c / 2}^{c / 2} \exp \left(i \alpha_{n} x\right) \sin \left(\frac{m \pi x}{c}\right) \mathrm{d} x, \quad \text { if } m \text { is even, } \\
& J_{m n}=\frac{1}{c} \int_{-c / 2}^{c / 2} \exp \left(i \alpha_{n} x\right) \cos \left(\frac{m \pi x}{c}\right) \mathrm{d} x, \quad \text { if } m \text { is odd. }
\end{aligned}
$$

The projection of $\mathrm{Eq}$. (A2) over the orthogonal set $\left\{\exp \left(i \alpha_{n} x\right),-d / 2 \leq x \leq d / 2\right\}$ leads to

$$
\begin{aligned}
& \frac{1}{d} \int_{-d / 2}^{d / 2} E_{R}\left(x, 0^{+}\right) \exp \left(-i \alpha_{n} x\right) \mathrm{d} x \\
& \quad=\frac{1}{d} \int_{-c / 2}^{c / 2} E_{M}\left(x, 0^{-}\right) \exp \left(-i \alpha_{n} x\right) \mathrm{d} x,
\end{aligned}
$$

which results in

$$
A_{n}=-\delta_{n, 1}+\frac{c}{d} \sum_{m=1}^{\infty} J_{m n}^{*} \sin \left(\mu_{m} h\right) a_{m} .
$$

The coupled system (A3)-(A6) can be solved to calculate the amplitudes $\left(A_{0}, A_{-1}, A_{1}, \ldots\right)$ and $\left(a_{1}, a_{2}, \ldots\right)$. Alternatively, Eqs. (A3) and (A6) can be combined to obtain the matrix equation

$$
\mathbf{G a}=\mathbf{H} \leftrightarrow \sum_{q=1}^{\infty} G_{m q} a_{q}=H_{m}, \quad m=1,2, \ldots,
$$

with the modal amplitudes as unknowns. The elements of the $\mathbf{G}$ matrix and $\mathbf{H}$ right hand side vector read

$$
\begin{gathered}
G_{m q q}=\frac{1}{2} \delta_{m, q} \mu_{q} \cos \left(\mu_{q} h\right)-\frac{c}{d} \sin \left(\mu_{q} h\right) \sum_{n=-\infty}^{\infty} i \chi_{n} J_{m n} J_{q n}^{*}, \\
H_{m}=-2 i \chi_{((n)} J_{m i 1)} \quad m=1,2, \ldots
\end{gathered}
$$

If the conditions for operation in the monomode regime are ensured [[14], Section 12], then the amplitude of the fundamental mode can be straightforwardly expressed as

$$
a_{1}=\frac{H_{1}}{G_{11}}=\frac{-2 i \chi_{10} J_{10}}{\frac{1}{2} \mu_{1} \cos \left(\mu_{1} h\right)-\frac{c}{d} \sin \left(\mu_{1} h\right) \sum_{n=-\infty}^{\infty} i \chi_{n}\left|J_{1 n}\right|^{2}} .
$$

The resonance wavelengths can be found by seeking the roots of the denominator in $\mathrm{Eq}$. (A8). However, as appointed in [14], the roots can be calculated with good approximation if only the terms $n=-1,0$, and 1 are retained. The discussion is further simplified by assuming that (1) the incident angle is $\theta=0^{\circ}$ and (2) only the zeroth reflected order is propagative. Equation (A8) then reads

$$
a_{1}=-\frac{\left.4 i \chi_{(10} J_{10}\right) \cos \left(\mu_{1} h\right)}{\mu_{1}-2 i \frac{c}{d} \chi_{(1)} \tan \left(\mu_{1} h\right)\left|J_{10}\right|^{2}+4 \frac{c}{d}\left|\chi_{1}\right| \tan \left(\mu_{1} h\right)\left|J_{11}\right|^{2}} .
$$

Therefore, we must find the roots of

$$
D^{R}(\lambda)=\mu_{1}(\lambda)+4 \frac{c}{d}\left|\chi_{1}(\lambda)\right|\left|J_{11}(\lambda)\right|^{2} \tan \left[\mu_{1}(\lambda) h\right],
$$

$$
D^{I}(\lambda)=2 \frac{c}{d} \chi_{0}(\lambda)\left|J_{10}(\lambda)\right|^{2} \tan \left[\mu_{1}(\lambda) h\right] .
$$

The roots $\lambda_{j}^{I}$ of $\mathrm{Eq}$. (All) are

$$
\lambda_{j}^{I}=2 \nu_{1}\left(j^{2} / h^{2}+1 / c^{2}\right)^{-1 / 2}, \quad j=1,2, \ldots .
$$

Finally, we can evaluate the magnitude of the monomodal field inside the grooves at each quasi-resonance wavelength $\lambda_{j}^{I}$ as

$$
\begin{aligned}
|E(0, y)|_{\lambda=\lambda_{j}^{l}} & =\left|a_{1} \sin \left[\mu_{1}\left(\lambda_{j}^{I}\right)(y+h)\right]\right| \\
& =\frac{8 \chi_{0}}{\pi}\left|\frac{\sin \left[\mu_{1}\left(\lambda_{j}^{I}\right)(y+h)\right]}{\mu_{1}\left(\lambda_{j}^{I}\right)}\right| .
\end{aligned}
$$

\section{ACKNOWLEDGMENTS}

This work has been supported in part by the European Union (EU) Program IBPOWER (211640) and the NUMANCIA II project (funded by Comunidad de Madrid).

\section{REFERENCES}

1. A. Luque and A. Martí, "The intermediate band solar cell: Progress toward the realization of an attractive concept," Adv. Mater. 21, 1-15 (2010).

2. B. Sopori, "Thin film silicon solar cells," in Handbook of Photovoltaic Science and Technology, A. Luque and S. Hegedus, eds. (Wiley, 2004), pp. 307-358.

3. H. Shpaisman, O. Niitsoo, I. Lubomirsky, and D. Cahen, "Can up- and down-conversion and multi-exciton generation improve photovoltaics?" Sol. Energy Mater. Sol. Cells 92, 1541-1546 (2008)

4. E. Yablonovith and G. D. Cody, "Intensity enhancement in textured optical sheets for solar cells," IEEE Trans. Electron Devices ED-29, 300-305 (1982).

5. J. M. Rodríguez, I. Tobías, and A. Luque, "Random pyramidal texture modelling," Sol. Energy Mater. Sol. Cells 45, 241-258 (1997).

6. F. Cajko and I. Fedoseyev, "Photoabsorption and carrier transport modeling in thin multilayer photovoltaic cell," Lect. Notes Comput. Sci. 5544, 755-764 (2009)

7. K. H. Brenner, "Aspects for calculating local absorption with the rigorous coupled-wave method," Opt. Express 18, $10869-10376$ (2010).

8. M. J. Mendes, A. Luque, I. Tobías, and A. Martí, "Plasmonic light enhancement in the near-field of metallic nanospheroids for application in intermediate band solar cells," Appl. Phys. Lett. 95, 071105 (2009).

9. K. R. Catchpole and A. Polman, "Plasmonic solar cells," Opt. Express 16, 21793-21800 (2008).

10. S. A. Maier, Plasmonics: Fundamentals and Applications (Springer, 2008).

11. P. Hewageegana and V. Apalkov, "Quantum dot photodetec- 
tors with metallic diffraction grating. Surface plasmons and strong absorption enhancement," Physica E (Amsterdam) 40, 2817-2822 (2008).

12. J. R. Andrewartha, J. R. Fox, and I. J. Wilson, "Resonance anomalies in the lamellar grating," Opt. Acta 26, 69-89 (1979).

13. A. Wirgin and A. A. Maradudin, "Resonant enhancement of the electric field in the grooves of bare metallic gratings $\mathrm{ex}$ posed to S-polarized light," Phys. Rev. B 31, 5573-5576 (1985).

14. A. Wirgin and A. A. Maradudin, "Resonant response of a bare metallic grating to S-polarized light.," Prog. Surf. Sci. 22, 1-99 (1986)

15. A. Barbara, P. Quémerais, E. Bustarret, T. Lopez-Ríos, and
T. Fournier, "Electromagnetic resonances of sub wavelength rectangular metallic gratings," Eur. Phys. J. D 23, 143-154 (2003).

16. T. López-Rios, D. Mendoza, F. J. Garcia-Vidal, J. SánchezDehesa, and B. Pannetier, "Surface shape resonances in lamellar metallic gratings," Phys. Rev. Lett. 81, 665-668 (1998).

17. F. J. Garcia-Vidal, J. Sanchez-Dehesa, A. Dechelette, E. Bustarret, T. López-Ríos, T. Fournier, and B. Pannetier "Localized surface plasmons in lamellar metallic gratings," J. Lightwave Technol. 17, 2191-2 195 (1999).

18. R. Petit, ed., Electromagnetic Theory of Glatings (SpringerVerlag, 1980).

19. J. A. Kong, Electromagnetic Wave Theory (Wiley, 1986). 